\title{
SOUTHERN ELEPHANT SEALS AS SUBJECTS FOR PHYSIOLOGICAL RESEARCH
}

\author{
by M.M. Bryden
}

(with one text-figure and two plates)

BRYDEN, M.M., 1988 (viii): Southern elephant seals as subjects for physiological research. Pap. Proc. Roy. Soc. Tasm., 122(1): 153-157, 2 pl. Papers presented at the Macquarie Island Symposium, Hobart, May 1987. https://doi.org/10.26749/rstpp.122.1.153 IS SN 0080 -4703. Formerly University of Queensland, now Department of Veterinary Anatomy, University of Sydney, New South Wales, Australia 2006.

Anatomical and physiological studies of southern elephant seals (Mirounga leonina), particularly in the post-natal period, raise questions of relative musculature growth, control of metabolism, circulation and temperature regulation, which could be important in our understanding of these processes in mammals and of their contribution to adaptation to environmental extremes.

Key words: elephant seals, marine mammals, Macquarie Island.

\section{INTRODUCTION}

Elephant seals, like other southern phocid seal species, are of great interest physiologically. As mammals that live protracted periods in the sea, they are subjected to certain physiological extremes not experienced by terrestrial mammals. For example, in diving for food, they experience rapid and great changes in pressure, and have developed means of breath-holding for long periods (in excess of one hour); similarly, in an environment in which heat loss from the body surface may be maximal, they must be able to control body temperature.

Since seals are amphibious, adaptation to the aquatic environment is different from that in totally aquatic species, such as whales. While making anatomical and physiological adaptations for life in the sea, seals must retain the ability to cope with living on land. The aim of this paper is to highlight a few of these special adaptations, using findings from the investigations of a research group over several years. Two major areas will be addressed, namely growth and development, and the regulation of body temperature. The paper is essentially a review and more details can be found in the references cited.

\section{GROWTH OF BODY COMPONENTS}

Elephant seals are born on land, and in certain respects they appear to be terrestrial mammals that develop their aquatic modifications postnatally. A body component vital for survival in cold water, but lacking at birth, is fat. In these seals, as in all other marine mammals, a large proportion of the body fat is deposited in the subcutaneous blubber layer which acts as essential insulation (Bryden 1964).

\section{Lactation and Blubber}

Lactation is brief in elephant seals (approximately three weeks) and growth is necessarily rapid, pups increase three- to four-fold on their birth weight in this period. Plates 1 and 2 illustrate the appearance of the pup at birth and at three weeks of age. Figure 1 shows that a large proportion of this body weight increase is blubber, but some growth of the musculature and other body systems occurs as well (Bryden 1969).

\section{Muscles}

Growth occurs at different relative rates among various muscle groups, favouring those important for terrestrial locomotion just after birth, and those important for swimming later (Bryden 1969). The pattern of development of the musculature is centripetal during the first weeks of postnatal life (the "terrestrial phase"). Growth spreads from the limbs and the head towards the body; the muscles surrounding the spinal column and those of the abdominal wall develop later in this phase of growth. However, these relative growth changes are reversed during the postweaning fast, when the animal loses body weight significantly.

A considerable change in growth patterns occurs when the seals enter the sea and swim extensively (the "marine phase"). Then relative 


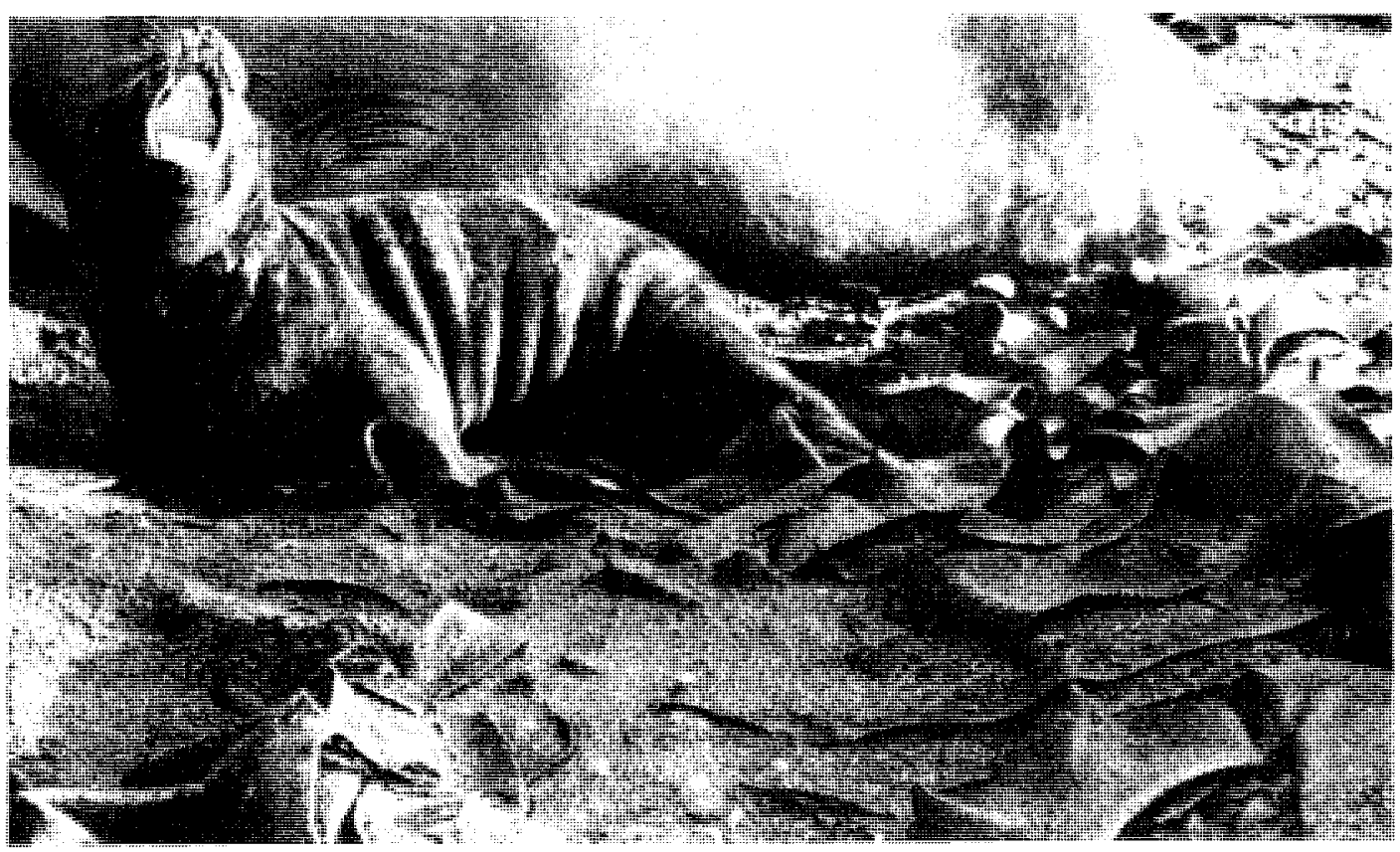

\section{PLATE 1}

Day-old elephant seal pup and its mother. Note relative size of adult female and pup.

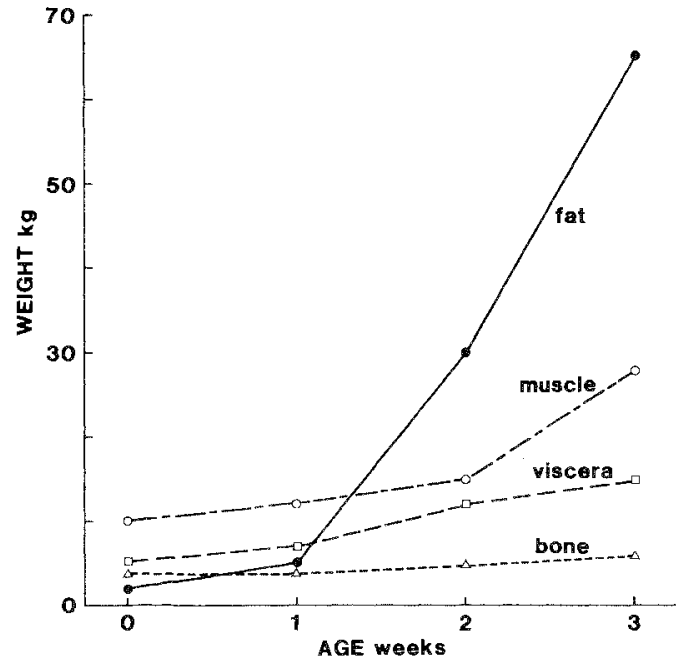

FIG. I - The increase in weight of four major body components of the elephant seal from birth to three weeks of age. growth favours those muscle groups used extensively in aquatic locomotion, in particular the muscles surrounding the spinal column and the proximal muscles of the hind limb.

This evidence of alteration in the relative size of muscles, as a result of changes in the functional demands placed on them, has important implications in an area quite remote from seal biology the study of methods used to improve meat production and quality.

\section{Circulatory System}

The development of the circulatory system reflects the change in the body's demand for oxygen when the seal first enters the sea and begins to dive (Bryden \& $\lim 1969$ ); specifically, the amount of blood in the system increases from about $11 \%$ of body weight at birth to $16 \%$ in animals eight months old or older. Changes in blood volume, red cell count and haematocrit occur when the young seal goes to sea. Bryden and Lim estimated that the greater oxygen capacity of the blood would allow the animal to remain submerged 3.5 times longer than a terrestrial mammal, without recourse to other physiological adjustments. 


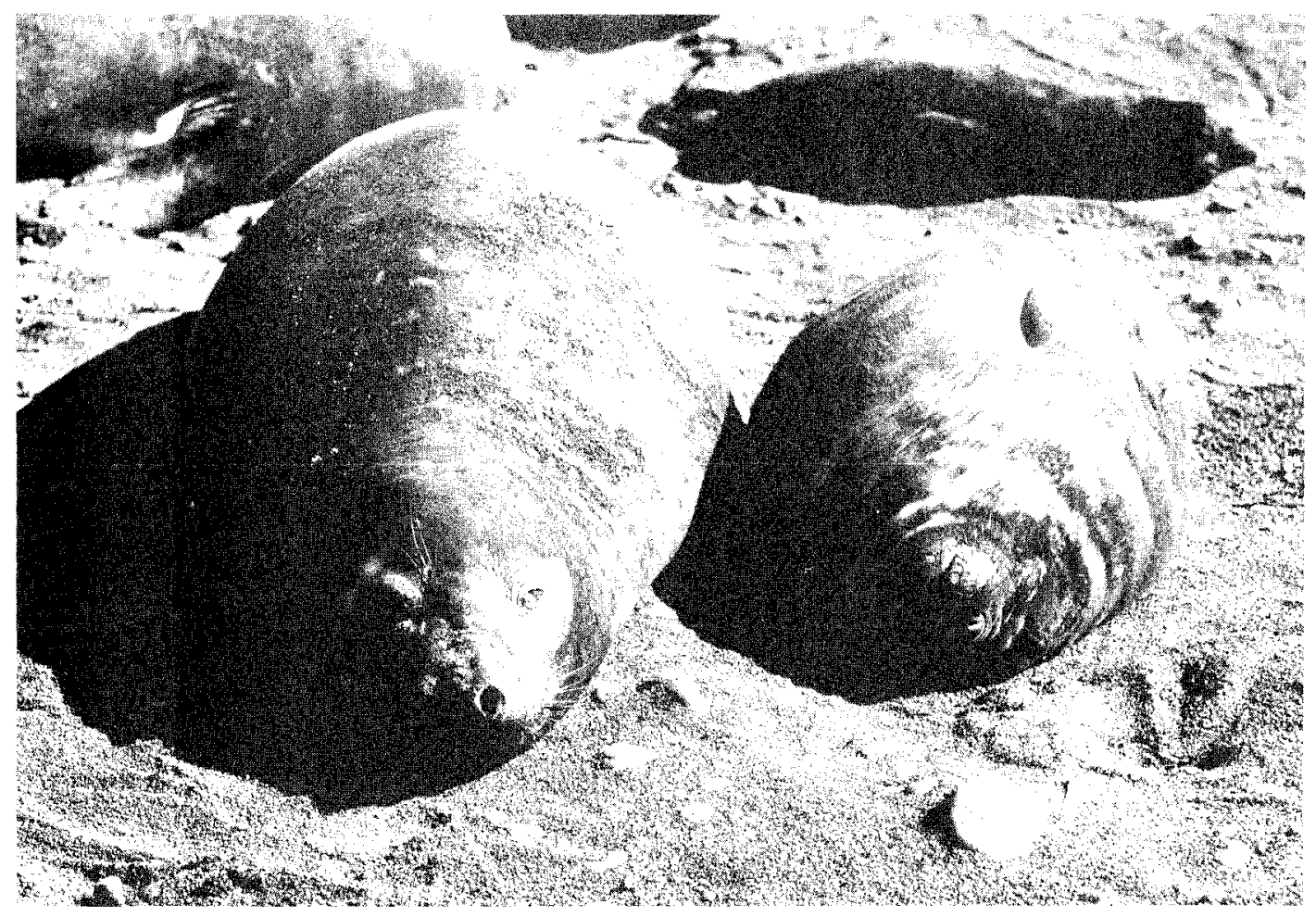

PLATE 2

Twenty-one-day-old elephant seal pup and its mother. A younger pup is in the background.

\section{REGULATION OF BODY TEMPERATURE}

\section{The First Hours of Postnatal Life}

Elephant seals are born into what, for a mammal at least, is a hostile environment. They possess little physical insulation at birth; they are wet, from amniotic fluid, and may remain so for long periods, since rain, sleet or snow is common. Although temperatures are not extreme, they are frequently near freezing with, in addition, a significant wind-chill factor. Pups are afforded no protection by the mother and exhibit no behavioural characteristics that would aid retention of body heat, such as huddling against the mother, curling up to reduce body surface area, or seeking protection from wind and precipitation.

Although Laws (1953) stated that elephant seals may be partially ectothermic in the first days postpartum, this was not confirmed in recent investigations (G.J. Little, pers. comm.) which suggest strongly that pups are endothermic from the time of birth, and low body temperature is indicative of cold stress.

\section{Vascular System}

Newborn elephant seals possess the structures required for fine control of the microcirculation in the skin and used in the regulation of body temperature; the arrangement of the capillaries and, notably, the density and distribution of arteriovenous anastomoses are the same in newborn and adult seals (Molyneux \& Bryden 1978).

The role of arteriovenous anastomoses in the peripheral circulation is, briefly, to permit arterial blood to be shunted directly into the venous system, bypassing the capillary bed (Molyneux \& Bryden 1981). When the anastomoses are open, large volumes of blood are passed from arteries to superficial veins; thus the rate of perfusion of the skin by blood is increased considerably, resulting in greater heat loss from the skin surface.

As an indication of the importance of this mechanism in the control of body temperature, it 